\title{
Likely Residual Metabolic Tumor Activity by PET
}

National Cancer Institute

\section{Source}

National Cancer Institute. Likely Residual Metabolic Tumor Activity by PET. NCI

Thesaurus. Code C116098.

A finding of focal metabolic activity at the primary or nodal sites that is greater than liver uptake, as detected by FDG-PET. 